\title{
Prevalência e fatores associados a sobrepeso e obesidade em escolares da rede pública
}

\author{
Prevalence and factors associated to overweight and obesity \\ in public schools
}

Paula Afonso Rodrigues ${ }^{1}$

$M$ aria Helena $M$ arques $^{2}$

$M$ aria das Graças Afonso M iranda Chaves $^{3}$

Camila Freitas de Souza ${ }^{4}$

M atheus Furtado de Carvalho ${ }^{5}$

${ }^{1}$ FaculdadedeM edicina, UniversidadeFederal deJuiz deFora. CampusdaUFJF, Martelos. 36016-970 Juiz de Fora M G.

paularodriguesmed@

yahoo.com.br

${ }^{2}$ Departamento deM edicina

Preventiva, Faculdade de

M edicina, Universidade

Federal de uiz deFora.

${ }^{3}$ Departamento de

Patologia, Faculdadede

Odontologia, Universidade

Federal deJuiz deFora.

${ }^{4}$ Faculdadede M edicina,

UniversidadeFederal de

Juiz deFora.

${ }^{5}$ Faculdade de O dontologia,

UniversidadeFederal de

Juiz deFora.
Abstract This study assessed the association be tween environmental factors and the prevalence of overweight and obesity in public schools. It was studied 480 students, by means of anthropometric measurements evaluation, weight and height BMI by sex and age and in accordance with the parameters of WHO and IOTF. Environmental information was obtained using a questionnaire applied to students. The Epi Info Program, version 3.3.2, was used to data collection and analysis. Prevalence rates of obesity varied according to the criteria it was used. According to the IOTF, prevalence of obesity was $7.1 \%$, while, according parameters of the $\mathrm{WHO}$, the prevalence was $18 \%$. The significant risk factor for obesity was the father obesity ( $P R=1.08 ; \mathrm{Cl}: 0.57-2.04)$. We concluded that the nutritional profile of the school servesasa subsidy for tracking trends of overweight in this group. This study could instruct the rationalization of the use of the time and public financial resources, proving the need to invest in the school feeding and in the health students.

Key words School feeding, O besity, Overweight, Students, Anthropometry
Resumo 0 objetivo do estudo foi verificar a pre valência de sobrepeso e obesidade em escolares e sua associação com variáveis ambientais. Foram estudados 480 alunos da rede pública, através de avaliação antropométrica, peso e altura (IMC), segundo sexo e idade e de acordo com os parâmetros da O M S eIOTF. Os fatores ambientais foram obtidos a partir de questionário aplicado junto aos escolares. 0 programa Epi Info, versão 3.3.2, foi utilizado para entrada eanálise dos dados. A prevalência de obesidade varia consideravelmente conforme o critério usado. Segundo parâmetros da IOTF, $7,1 \%$ dos escolares foram considerados obeSos, ao passo que, deacordo com critérios da OM S, a prevalência deobesidadefoi igual a 18\% . 0 fator de risco significante para a obesidade da criança e adolescente foi apenas a obesidade do pai ( $R P=$ $1,08 ;$ IC: $0,57-2,04)$. 0 perfil nutricional dos escolares serve como subsídio para o monitoramento de tendências de sobrepeso nesse grupo e para a elaboração de medidas preventivas.

Palavras-chave Alimentação escolar, 0 besidade, Sobrepeso, Estudantes, Antropometria 


\section{Introdução}

O Brasil tem apresentado profundas modificações no perfil nutricional de sua população, fruto de um processo conhecido como transição nutricional ${ }^{1}$. Além da tendência declinantededesnutrição, destacam-se prevalências relativamente baixas e estáveis de sobrepeso e obesidade entre menores de cinco anos, situando-seem torno de $5 \%{ }^{2}$. Em contraposição, em crianças de seis a dez anos e nos adolescentes, pode-se considerar que o sobrepeso éalteração nutricional em ascensão.

No Canadá, Austrália e parte da Europa, as taxas de aumento de excesso de peso na população infantil, na década de noventa, alcançaram $1 \%$ ao ano ${ }^{3}$. No Chile, entre 1987 e 2000, em crianças deseisanos, verificou-se prevalência de $12 \%$ para $26 \%$ para os meninos e de $14 \%$ para $27 \%$ em meninas; na China, a prevalência desobrepeso e obesidade em crianças dessa mesma idade aumentou de 7,7\% para $12,4 \%$ em um período de tempo bastante reduzido, entre 1991 e $1997^{4}$.

A obesidadeéuma condição multifatorial, com componente genético eambiental. 0 acúmulo de gordura corporal, sobretudo do tipo andróide presente na idade escolar, que persiste na adolescência, exerceefeitosfisiológicosepatológicoscom claros efeitos sobre a mortalidade e morbidade navida adulta. Além dos efeitos deletérios sobrea saúde, a obesidade se associa a problemas psiquiátricos como a depressão, a perda da autoestima e a alteração da imagem corporal ${ }^{5}$.

As características como grupo de risco e as possibilidades de sucesso das ações a serem implementadas fazem com que as crianças e os adolescentes constituam um dos principais gruposalvo para estratégias de prevenção e controle do sobrepeso e da obesidade ${ }^{6}$.

A avaliação nutricional de populações, especialmenteatravés da realização deinquéritos nutricionais no ambienteescolar, éuma ferramenta de suma importância para compreensão do perfil nutricional dos estudantese, consequentemente, para a racionalização dos recursos financeiros destinados às instituições.

A escola, assim, torna-se um espaço atrativo para avaliar a ocorrência de excesso de peso em crianças e adolescentes, visto a cobertura elevada do sistema escolar entre os estudantes desde 0 pré-escolar e a facilidade de obtenção de informações sobre o aluno devido ao apoio da rede escolar ${ }^{4}$.

Historicamente, embora sejam registradas ações governamentais na área de alimentação e nutrição já na década de trinta, estudos avaliati- vos dos programas de nutrição são escassos e dados populacionais sobre acesso a ações públicas nessa área carecem de atualização, principalmente em sua correlação com o estado nutricional dos grupos atendidos?.

0 presente estudo tem por objetivo verificar a prevalência de obesidade esobrepeso em escolares associada a fatores ambientais relacionados à atividade física, excesso de peso dos pais e alimentação escolar.

\section{Material e métodos}

Um estudo observacional foi realizado em escolas da rede pública da cidade de Juiz de Fora (M G), segundo listagem fornecida pela Superintendência Regional de Ensino - Juiz de Fora (SEDINE). As escolas possuem alunos de baixo a médio nível socioeconômico. 0 tamanho da amostra, 480 escolares de ambos os sexos, de quatro a dezoito anos de idade, foi definido seguindo as regras do Informe Técnico № 25 da Secretaria Estadual de Saúde de M inas Gerais $(\mathrm{SES})^{8}$. A coleta de dados foi realizada no período entre junho e novembro de 2006.

Inicialmente, foi sorteada a amostra das escolas públicas (municipais e estaduais) representativa da educação infantil/classe de alfabetização e primeiro e segundo segmentos do ensino fundamental ( $1^{\mathrm{a}}$ a $4^{\mathrm{a}}$ e $5^{\mathrm{a}}$ a $8^{\mathrm{a}}$ séries, respectivamente), totalizando vinte escolas escolhidas. Foi realizado o sorteio das turmas e, nas turmas, sorteio dos escolares. Em escolas que não possuíam alunos em todas as faixas etárias, examinamoso número de escolares quefaltava para completar a amostra por idade em outra escola. Os sorteios seguiram o padrão de amostragem aleatória simples.

As informações tais como data de nascimento e sexo foram obtidas das fichas de matrícula dos alunos. Um questionário, respondido individualmente pelos escolares, foi elaborado para atender aos objetivos desta pesquisa e possuía perguntas a respeito de seus hábitos alimentares, como a frequência com a qual a criança compra lanches na escola, considerando opções de uma vez a cinco vezes eincluindo as opções não compra lanchena escola ea escola não possui cantina. 0 uso semanal da alimentação escolar foi avaliado através de frequências que incluíam as opções de uma vez a cinco vezes ea alternativa nunca. 0 número de refeições diárias, considerando aque las realizadas na escola e na residência, foi avaliado segundo as opções menos de três, quatro, cin- 
co emais de cinco refeições. Em relação à atividade física dos escolares, foi perguntado se eles se consideravam sedentários, moderadamente ativos ou ativos. Já a obesidade dos pais foi analisada segundo a opinião dos filhos em relação aos pais, sendo que no questionário existiam as seguintes alternativas: minha mãeé obesa, meu pai é obeso, meus pais são obesos e meus pais não são obesos. As crianças menores respondiam o questionário com ajuda de cartilhas que demonstravam figuras referentes às perguntas.

Os escolares foram pesados e medidos, em dia agendado com a direção da escola, por uma equipe de alunos de graduação da área de saúde, previamente treinada pelos pesquisadores do projeto.

A avaliação antropométrica foi realizada seguindo padronização de Lohman et al. ${ }^{9}$. Para o registro do peso e altura, foram utilizadas balanças eletrônicas portáteis G-TECH FLAT II, com capacidade de $150 \mathrm{Kg}$, divisão de $100 \mathrm{gr}$ e um estadiômetro portátil, com escala de 35 a $213 \mathrm{~cm}$ e resolução de $0,5 \mathrm{~cm}$.

As medidas foram coletadas e registradas em duplicata sem arredondamentos. 0 índice de massa corporal (IMC) foi calculado como a razão da massa corporal $(\mathrm{kg})$ pelo quadrado da estatura $\left(m^{2}\right)$.

Devido à falta de consenso internacional sobre quais os melhores critérios antropométricos a serem adotados para avaliação de sobrepeso e obesidade em crianças e adolescentes, e visando facilitar a comparação dos resultados encontrados com estudos que utilizem outros critérios para diagnóstico de sobrepeso e obesidade, utilizou-se o IMC para a idade e sexo, considerando abaixo do percentil 5 (P5) como critério para baixo peso, IMC > percentil 85 (P85) como sobrepeso e percentil 95 (P95) do IM C como obeso, tendo como referência os valores da população americana ${ }^{10}$. Apresentam-se, também, no estudo, as prevalências de obesidade e sobrepeso segundo critério proposto pela International $O$ besity Task Force (IOTF) ${ }^{11}$.

Em uma ficha clínicaúnica eindividual, reuniram-se os dados adquiridos, que foram submetidos à análise estatística através do programa Epi Info, versão 3.3.2.

Asvariáveis consideradas como possivelmente associadas ao sobrepeso em adolescentes são padrão de atividade física, obesidade dos pais, frequência de uso da merenda escolar efrequência de compra de lanches em cantinas particulares. Esses fatores foram analisados individualmenteem relação ao estado nutricional. As variá- veis com associação significante foram aquelas $\operatorname{com} p<=0,05$.

0 testequi quadrado foi aplicado com nível de significância de 0,05 para as variáveis categóricas. 0 odds ratio (OR) com intervalo de confiança de $95 \%$ foi também calculado para as associações.

Adotou-se no estudo o termo "sobrepeso" para identificar crianças e adolescentes com sobrepeso bem como obesidade, constituindo-se a variável desfecho do estudo.

A pesquisa foi realizada dentro dos parâmetros contidos na Resolução n 196/96 do ConseIho Nacional de Saúde/M inistério da Saúde, que dispõe sobre pesquisas envolvendo seres humanos. Acredita-se quese cumpriu o que estabelece a referida resolução no momento em que se solicitou, junto às escolas, autorização para a realização do estudo e, dos sujeitos e pais, consentimento para o (a) aluno (a) participar da pesquisa, prestando-Ihes esclarecimentos sobre o objetivo do estudo, o seu direito de abandoná-lo e o do anonimato.

O Comitê de Ética em Pesquisa da Universidade Federal de Juiz de Fora aprovou o protocolo da pesquisa no dia 19 de janeiro de 2006.

\section{Resultados}

A amostra do estudo foi integrada por $241 \mathrm{mu}$ Iheres $(50,2 \%)$ e 239 homens (49,8\%), cuja mé dia de idade foi igual a $10,8 \pm 3,38$ anos. Os dados antropométricos revelaram média de $1,43 \pm 0,19$ metros para a al tura e $37,97 \pm 15,67$ quilos para o peso dos escolares.

A prevalência de sobrepeso e obesidade em crianças e adolescentes varia segundo os critérios usados. De acordo com os parâmetros da IOTF, para todos escolares, identificou-se um total de $12,5 \%$ e $11,8 \%$ para sobrepeso em meninas e meninos, respectivamente, e $4,2 \%$ e $2,9 \%$ para obesidade para os respectivos sexos, ao passo que a prevalência de sobrepeso, segundo a OMS, foi de $7,5 \%$ e $13,1 \%$ para o sexo feminino emasculino, respectivamente. Já os valores referentes à obesidadeforam de $10,4 \%$ entremeninase $7,6 \%$ entre meninos. $N$ ão foi observado um padrão de associação para mulheres (Gráfico 1) e homens (Gráfico 2) na prevalência de sobrepeso e obesidade de acordo com a idade.

A prevalência de desnutrição, até dez anos de idade, por déficit de massa corporal/estatura foi $5,3 \%$ e $6,5 \%$ para meninas e meninos, respectivamente, segundo critérios da OMS. Para escolares com idade maior que dez anos, a propor- 
ção de baixo peso foi de $14,3 \%$ entre meninos e $5,5 \%$ entre meninas, quando o esperado era $5 \%$, de acordo com o ponto de corte adotado (IM C $<$ percentil 5- P5).

$\mathrm{Na}$ análise dos fatores de risco para a obesidade da criança e adolescente, apenas a obesidade do pai demonstrou-se ser significativa. Como o nível de significância utilizado para selecionar possíveis fatores de risco foi inferior a $5 \%$, todos os intervalos de confiançaincluem a unidade. Em relação à prática deatividadefísica, comprar lanche na escola, número de refeições diárias e frequência de uso da merenda escolar, os adolescentes com IMC normal e os com sobrepeso se distribuíram de forma bastante próxima. $\mathrm{Ne}$ nhum dos parâmetros avaliados mostrou associação significativa (Tabela 1).

Dados do estudo revelaram que $47,1 \%$ dos alunos entrevistados desconhecem a obesidade como doença.

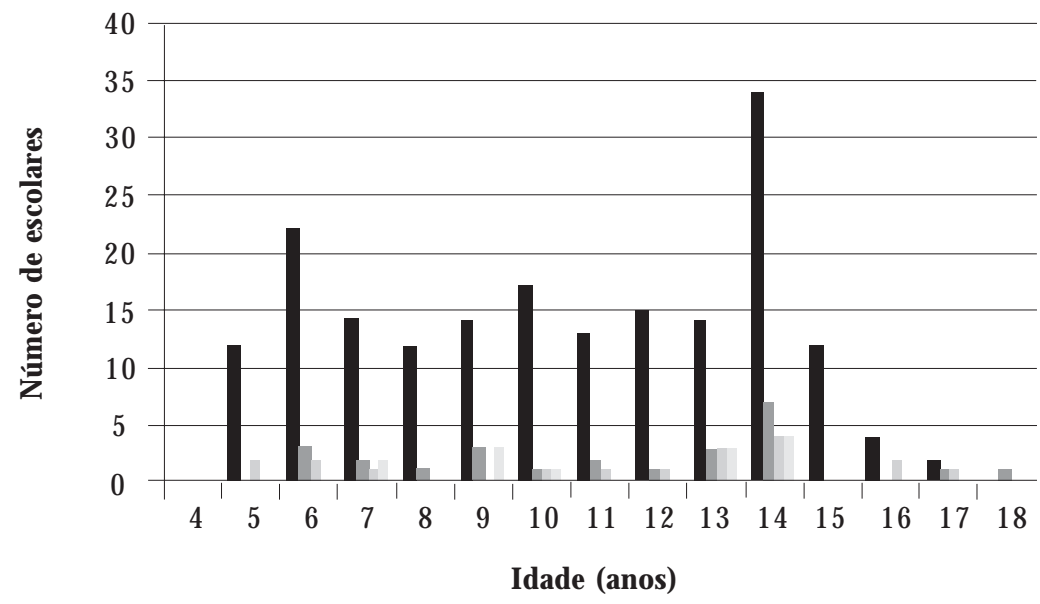

Normal

Obeso

Sobrepeso

Baixo peso

Figura 1. Distribuição de escolares do sexo feminino, de acordo com critérios da OM S, segundo idade. Juiz de Fora, MG, 2006.

$$
\begin{aligned}
& y \\
& \frac{1}{0} \\
& \frac{0}{0} \\
& y \\
& 0 \\
& 0 \\
& 0 \\
& \frac{1}{2} \\
& \frac{8}{2} \\
& 2
\end{aligned}
$$

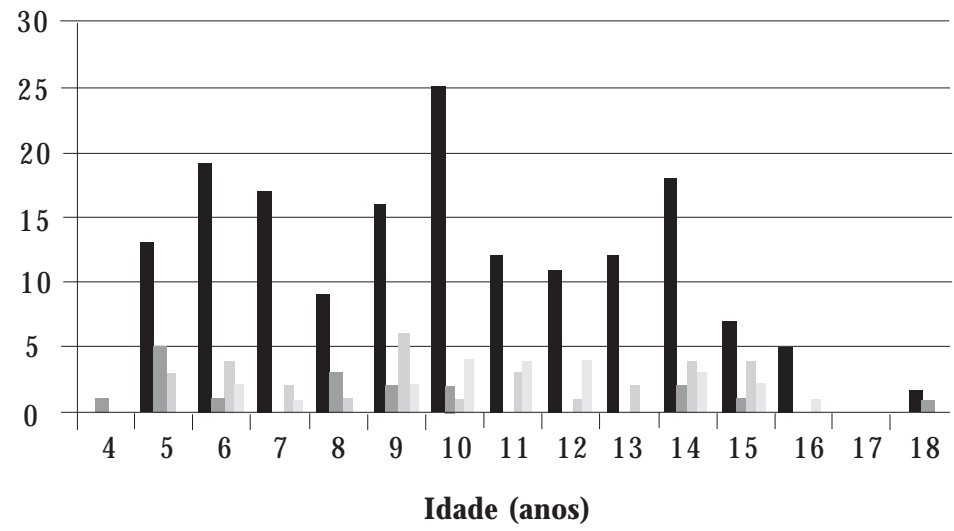

Normal

Obeso

Sobrepeso

Baixo peso

Figura 2. Distribuição de escolares do sexo masculino, de acordo com critérios da OMS, segundo idade. Juiz de Fora, MG, 2006. 
Tabela 1. Prevalência (\%) e odds ratio de sobrepeso em escolares do ensino fundamental, de acordo com as variáveis ambientais.

\begin{tabular}{|c|c|c|c|c|}
\hline & Percentual & Odds & IC 95\% & $p^{*}$ \\
\hline Costuma comprar lanche na escola & & & & 0,985 \\
\hline Uma vez & $23,1 \%$ & 0,97 & $0,56-1,67$ & \\
\hline Duas vezes & $10,8 \%$ & 0,85 & $0,40-1,82$ & \\
\hline Três vezes & $5,0 \%$ & 0,58 & $0,17-1,98$ & \\
\hline Quatro vezes & $2,1 \%$ & 1,04 & $0,22-4,97$ & \\
\hline Cinco vezes & $5,8 \%$ & 1,14 & $0,45-2,90$ & \\
\hline Não & $22,7 \%$ & 1,10 & $0,64-1,88$ & \\
\hline A escola não tem cantina & $27,3 \%$ & 1,11 & $0,67-1,84$ & \\
\hline $\begin{array}{l}\text { Quantas vezes por semana faz uso } \\
\text { da merenda escolar }\end{array}$ & & & & 0,663 \\
\hline Uma vez & $11,3 \%$ & 1,96 & $1,02-9,75$ & \\
\hline Duas vezes & $10,8 \%$ & 0,85 & $0,40-1,82$ & \\
\hline Três vezes & $13,8 \%$ & 0,63 & $0,30-1,32$ & \\
\hline Quatro vezes & $5,2 \%$ & 1,05 & $0,38-2,88$ & \\
\hline Sempre & $26,7 \%$ & 0,89 & $0,53-1,50$ & \\
\hline Nunca & $32,3 \%$ & 0,98 & $0,60-1,61$ & \\
\hline $\begin{array}{l}\text { Como você se considera em relação } \\
\text { à atividade física }\end{array}$ & & & & 0,781 \\
\hline Sedentário & $9,5 \%$ & 2,04 & $0,85-4,91$ & \\
\hline Ativo & $52,3 \%$ & 0,96 & $0,61-1,51$ & \\
\hline M oderadamente ativo & $38,2 \%$ & 0,90 & $0,57-1,43$ & \\
\hline Você considera seus pais obesos & & & & 0,031 \\
\hline Sim, meu pai & $14,5 \%$ & 1,08 & $0,57-2,04$ & \\
\hline Sim, minha mãe & $16,9 \%$ & 0,54 & $0,57-1,10$ & \\
\hline Sim, ambos & $4,2 \%$ & 0,90 & $0,12-7,02$ & \\
\hline Não & $64,4 \%$ & 1,57 & $0,94-2,63$ & \\
\hline Número de refeições diárias & & & & 0,699 \\
\hline M enos de três & $2,1 \%$ & 0,41 & $0,05-3,22$ & \\
\hline Três & $15,9 \%$ & 1,26 & $0,69-2,28$ & \\
\hline Quatro & $44,8 \%$ & 0,94 & $0,59-1,48$ & \\
\hline Cinco & $28,0 \%$ & 1,08 & $0,66-1,79$ & \\
\hline M ais de cinco & $9,2 \%$ & 0,93 & $0,42-207$ & \\
\hline
\end{tabular}

*associado ao teste $T$ de Student.

Em relação à merenda escolar, os resultados mostraram que ela era distribuída em todas as escolas, apresentando cardápios variados, cujos principais componentes são apresentados de acordo com Tabela 2. Uma vez por semana, era servida uma refeição doce, como arroz doce ou canjica. Cada escola apresentava um cardápio alimentar semanal próprio, que possuía componentes semelhantes entre elas, porém nem sempre dispostos nos mesmos dias da semana.

A alimentação oferecida pelas escolas apresentou índice de rejeição igual a 36,4\%. Além de não gostar $(17,9 \%)$, as razões que alunos mais citaram para não comerem a merenda escolar foram compra de lanche nas cantinas particulares $(14,2 \%)$ e não ter vontade ou fome para comer $(17,3 \%)$. A proximadamente $98 \%$ dos esco-
Tabela 2. Cardápio da merenda de uma das escolas participantes do estudo. Juiz de Fora (MG), abril de 2006.

\begin{tabular}{ll}
\hline Dia da semana & \multicolumn{1}{c}{ Cardápio } \\
\hline Segunda-feira & $\begin{array}{l}\text { Arroz temperado, frango, } \\
\text { cenoura e macarronada }\end{array}$ \\
Terça-feira & $\begin{array}{l}\text { Arroz, feijão e angu baiano } \\
\text { Quarta-feira }\end{array}$ \\
Quinta-feira & $\begin{array}{l}\text { Sopa de macarrão com salsinha, } \\
\text { batata-inglesa, cenoura e vagem }\end{array}$ \\
Sexta-feira & Arroz doce ou canjica
\end{tabular}

lares fazem mais detrês refeições por dia, incluindo aquelas realizadas em casa, sendo que apenas $22,9 \%$ dos entrevistados afirmam ter uma alimentação melhor na escola. 
A maior parte das escolas possuía cantinas $(70,0 \%)$, lanchonetes ou outros fornecedores de alimentos aos alunos, além da merenda escolar. Alimentos como biscoitos, doces, guloseimas e salgados caseiros eram os preferidos pelos escolares, apesar de $56,1 \%$ dos entrevistados avaliarem esses alimentos como não sendo melhores para saúde do que aqueles oferecidos pela merenda escolar.

\section{Discussão}

O espaço escolar é um local factível e oportuno para a obtenção de informações representativas locais sobre saúde e nutrição, pouco disponíveis para crianças maiores de cinco anos e adolescentes ${ }^{12}$. 0 presente estudo, por usar uma amostra probabilística, apresenta o perfil nutricional dos alunos do ensino público da cidadedeJuiz deFora.

A partir da notificação, em praticamentetodo o mundo, do aumento importante na magnitude de obesidade e sobrepeso em adultos, atenção passou a ser dada para a avaliação nutricional de crianças e adolescentes. 0 percentual de escolares de Juiz de Fora com IM C acima do percentil 95 é inferior em relação aos 15\% de obesos para ambos os sexos na população americana ${ }^{13}$.

A prevalência de obesidade e sobrepeso variou consideravelmente conformeo critério usado. Segundo os autores, os pontos de corte estabelecidos pelo IOTF são menos arbitrários, já que são baseados nos pontos de corte de IMC para adultos que melhor predizem morbidade e mortalidade. 0 diferencial deste padrão de referência éque os pontos de corte para classificação de sobrepeso e obesidade por sexo e faixa etária foram definidos através da interceptação, aos dezoito anos de idade, com os pontos de IMC já utilizados para classificação de sobrepeso ( $25 \mathrm{a}$ $30 \mathrm{~kg} / \mathrm{m} 2$ ) e obesidade (> $30 \mathrm{~kg} / \mathrm{m} 2$ ) em adultos e, a partir desses valores, foram construídas curvas para as idades menores de dezoito anos.

Segundo documento internacional sobreobesidade, as crianças têm mais chance de serem obesas em países ou regiões onde o processo de transição nutricional tem-se dado rapidamente, apresentando tendência secular positiva do crescimento linear e redução da prevalência do déficit de estatura ${ }^{14}$. Assim, é de se esperar que, seguindo esse ritmo, o mesmo fenômeno ocorra no Brasil, visto que, nas últimas décadas, tem sido registrada esta tendência no país. No período de 1974 a 1997, a prevalência de crianças e adolescentes obesos e com sobrepeso aumentou de $4,1 \%$ para $13,9 \%$, sendo o aumento ainda maior nas áreas urbanas do país e no nível socioeconômico mais elevado ${ }^{15}$.

A obesidade é o resultado da interação entre a susceptibilidade do hospedeiro eum meio ambiente que promova a doença. Crianças e adolescentes com alto I M C, geralmente, levam essa característica até a vida adulta, com conhecidas re percussões para a saúde e morbimortalidade ${ }^{16}$.

O consumo alimentar como um todo não tem sido consistentemente associado ao estado nutricional. Resultado de estudo, contrariamente à opinião popular, mostrou que não apenas um grupo alimentar individual, mas sim uma combinação de muitos alimentos, foi associada com excesso de adiposidade ${ }^{17}$. Segundo M affeis et al. ${ }^{18}$, apenas a composição da dieta não contribui para explicar a adiposidade em crianças quando o sobrepeso (IMC) dos pais foi avaliado.

No Sudeste, aproximadamente $94 \%$ do total de crianças matriculadas em escolas públicasfrequentam estabel ecimentos que oferecem refeição gratuita. A análise da oferta da alimentação escolar, segundo a vinculação do estabelecimento às redes federal, estadual e municipal de ensino, indica um papel expressivo das escolas estaduais na oferta de refeição ${ }^{19}$. Segundo o Programa Nacional de Alimentação Escolar (PNAE), a me renda escolar precisa garantir cerca de 350 quilocalorias e09 gramas de proteína. Entretanto, além de considerar o valor calórico das refeições, é preciso avaliar a adequação dos alimentos do PNAE às necessidades dos indivíduos.

Em 1992, o governo federal possibilitou maior adaptação dos cardápios à realidade cultural local e o consumo de frutas, verduras e legumes frescos, no lugar dos alimentos industrializados. Mas, de acordo com os diários das dietas escolares do estudo, foi possível observar uma merenda rica em carboidratos e açúcares e, portanto, com déficit nutricional. Desta forma, crianças e adolescentes que têm a merenda escolar como a principal refeição diária carecem de alimentação adequada e, portanto, observa-se uma proporção de escolares com baixo peso maior que 0 esperado segundo critérios da OMS.

0 índice de rejeição apresentado pelos escolares em relação à merenda escolar foi alto eacredita-se que tal fato deve-se ao aumento do consumo de lanches, considerado o maior observado na última década. A ingestão média de calorias provenientes dos lanches aumentou de 450 para 600 cal orias por dia e hoje representa $25 \%$ da ingestão energética diária. Este achado é importante, já que pequenas elevações na densidade ener- 
gética de alimentos consumidos podem levar a grande aumento na ingestão calórica total ${ }^{20}$.

A obesidade dos pais está fortemente associada à obesidade dos filhos, apresentando força de associação de magnitude expressiva (OR = 2,5). Esta relação tem sido bem descrita na literatura e envolve herança genética, fatores relacionados ao desenvolvimento de atitudes tendo os pais como referência e a decisão dos pais ou familiares sobre os hábitos da criança. Atitudes em relação à promoção de atividades de lazer podem influenciar o padrão de atividade física da criança $a^{21}$. Os dados da pesquisa revelaram associação positiva entre obesidade do pai e sobrepeso da criança ou adolescente.

No presente estudo, nenhuma diferença significante entre os indicadores que compuseram a avaliação da atividade física foi detectada comparando escolares que apresentavam sobrepeso com aqueles que não 0 apresentavam. O utros estudos indicaram correlações negativas entreIM C eexercícios ${ }^{22,23}$. A atividade física e/ou fatores metabólicos têm importância na manutenção da obesidade, ao passo que os fatores da dieta são mais importantes no início da obesidade, uma vez que a modificação na dieta é melhor preditor para a perda de peso do que uma mudança na atividade física enos fatores metabólicos ${ }^{24}$. Embora os exercícios físicos não estejam claramente associados ao sobrepeso decrianças eadolescentes, éimportante ressaltar seu papel no bem-estar social e, ainda na infância, em comportamentos como prevenção do tabagismo euso de drogas, estímulo à atividade sexual, à alimentação saudável e ao desempenho acadêmico 25 .

\section{Considerações finais}

Os resultados do presente estudo, ao identificar o perfil nutricional dos escolares do ensino público do município do Juiz deFora, servem como subsídio para o monitoramento de tendências de sobrepeso nesse grupo.

A proporção de crianças e adolescentes com sobrepeso é elevada e condiz com os resultados deoutros estudos realizados no país. 0 desenvolvimento de medidas de prevenção e controle do excesso de peso neste grupo etário deveria envolver não só a instituição escolar, através da elaboração de uma dieta equilibrada, mas também os pais ou responsáveis pelas crianças, uma vez que o ambiente familiar apresenta notável influência sobre a condição do sobrepeso infantil.

A valorização e o controle do crescimento físico na idade escolar éum aspecto relevantee de grande utilidade no controle da saúde dos escolares e do impacto da alimentação sobre seu desenvolvimento. Para este fim, a antropometria é um método de baixo custo, de técnica sensível e muito útil para o seguimento e o controle do estado nutricional dos escolares.

\section{Colaboradores}

PA Rodrigues, M H Marques, M GAM Chaves, CF Souza e MF Carvalho participaram igualmente de todas as etapas da elaboração do artigo. 


\section{Referências}

1. Popkin BM. The nutrition transition and obesity in the developing world. J Nutr. 2001; 131:871-873.

2. Monteiro CA, Conde $W L$, Popkin BM . A tendência secular da obesidade segundo estratos sociais: Nordeste e Sudeste do Brasil, 1975-1989-1997. Arq Bras Endocrinol M etab 1999; 43:186-194.

3. Lobstein T, Baur L, Uauy R. O besity in children and young people: a crisis in public health. 0 bes Rev. 2004; 5(1):4-85.

4. NSW Centre for Public Health Nutrition. Best options for promoting healthy weight and preventing weight gain in N SW. New South Wales: University of Sidney; 2005.

5. Poletti C, Oscar H, Barrios MI. Sobrepeso y obesidad como componentes de la malnutrición, en escolares de la ciudad de Corrientes: Argentina. Rev. Chil. Pediatr. 2003; 74(5):499-503.

6. Mondini L, Levy RB, Saldiva SRDM, Venâncio SI, Aguia JA, Stefanini M LR. Overweight, obesity and associated factors in first grade schoolchildren in a city of the metropolitan region of São Paulo, Brazil. Cad Saude Publica 2007; 23(8):1825-1834.

7. Valente $F$. Inserção dos componentes de alimentação e nutrição nas políticas governamentais e na estratégia internacional de desenvolvimento. Brasília: Organização das $\mathrm{N}$ ações Unidas para Agricultura e Alimentação;1996.

8. Secretaria Estadual de Saúde de M inas Gerais. [site da Internet] [acessado 2006 fev 02]. Disponível em: http://www.saude.mg.gov.br/

9. Lohman TG, Roche AF, M artorell R. Anthropometric Standardization Reference M anual. Champaign, Illinois: Human Kinetics; 1988.

10. World Health Organization. [site da Internet] [acessado 2006 out 28]. Disponível em: http://www.who. int/topics/obesity/en/

11. International O besity Taskforce. [site da Internet] [acessado 2006 dez 02]. Disponível em: http://www. iotf.org/

12. Anjos LA, Castro IRR de, Engstrom EM, Azevedo AM F. Crescimento e estado nutricional em amostra probabilística de escolares no município do Rio de Janeiro, 1999. Cad Saude Publica 2003; 19:171-179.

13. Ogden $\mathrm{CL}$, Flegal $\mathrm{KM}$, Carroll $M D$, Johnson $\mathrm{CL}$. Prevalence and trends in overweight among US children and adolescents, 1999-2000. JAM A 2002; 288:1728-1732.

14. Centers for Disease Control and Prevention. 2000 CDC Growth Charts for the U nited States: M ethods and Development. Vital Health Statistics Series 11. Washington, D.C.: U.S. Government Printing Office; 2002.
15. Lima SC, Arrais RF, Almeida M G, Souza ZM, Pedrosa LF. Plasma lipid profile and lipid peroxidation in overweight or obese children and adolescents. J. Pediatr. (Rio J.) 2004; 80(1):23-28.

16. Gill TP. Key issues in the prevention of obesity. $\mathrm{Br}$ Med Bull 1997; 53(2):359-388.

17. M ccarthy SN, Robson PJ, Livingstone MBE, Kiely M, Flynn A, Cran GW, Gibney M G. Associations between daily food intake and excess adiposity in Irish adults: towards the development of food-based dietary guidelines for reducing the prevalence of overweight and obesity. Int J O bes 2006; 30(6):993-1002.

18. Maffeis C, Provera S, Filippi L, Sidoti G, Schena S, Pinelli L, Tatò L. Distribution of food intake as a risk factor for childhood obesity. Int J O bes 2000; 24(1):75-80

19. Burlandy $L$, Anjos LA. Acesso à alimentação escolar e estado nutricional de escolares no Nordeste e Sudeste do Brasil, 1997. Cad Saude Publica 2007; 23(5):1217-1226.

20. Mello ED, Luft VC, Meyer F. Childhood obesity: towards effectiveness. J. Pediatr. (Rio J.) 2004; 80(3):173-182.

21. Ribeiro IC, Taddei JA, Colugnatti F. O besity among children attending elementary public schools in São Paulo, Brazil: a case-control study. Public Health Nutr 2003; 6(7):659-663.

22. Fonseca VM, Sichieri R, Veiga GV. Fatores associados à obesidade em adolescentes. Rev. Saude Publica 1998; 32(6):541-549.

23. M agalhães VC, Azevedo G, M endonça S. Prevalência e fatores associados a sobrepeso e obesidade em adolescentes de 15 a 19 anos das regiões Nordeste e Sudeste do Brasil, 1996 a 1997. Cad Saude Publica 2003; 19(Supl. 1):S129-S139.

24. Kiesges RC. Accuracy of self-reports of food intake in obese and normal-weight individuals: effects of parental obesity on reports of children's dietary intake. Am J Clin Nutr 1988;48:I252-6.

25. Goran MI, Reynolds KD, Lindquist $\mathrm{CH}$. Role of physical activity in the prevention of obesity in children. Int J Obes 1999; 23(3):18-33.

Artigo apresentado em 04/03/2008

Aprovado em 16/10/2008

Versão final apresentada em 03/12/2008 